\title{
Extension of TMZ maintenance therapy beyond six cycles: Two cases report with a good response
}

\author{
Ionela Bizu1, Cristina Tiut', Rodica Anghel 2,3
${ }^{1}$ Hospital OncoFort, Bucharest, Romania
2"Al. Trestioreanu" Institute of Oncology, Bucharest, Romania
3"Carol Davila" University of Medicine and Pharmacy, Bucharest, Romania
}

\begin{abstract}
Glioblastoma is an aggressive cerebral tumor characterized by increased morbidity and/or mortality. In 2005, concomitant chemoradiotherapy with temozolomide (TMZ), followed by adjuvant 6 cycles, became the standard-of-care therapy following tumor resection or biopsy.

We present two cases, newly diagnosed with glioblastoma, which received more than 6 maintenance cycles after surgical resection and radiotherapy with TMZ. In both cases, the drug was well tolerated. The optimal duration of maintenance therapy is still not clear. In both cases, the extended administration of TMZ therapy, had a significant activity and excellent tolerability.
\end{abstract}

Keywords: glioblastoma, temozolomide, adjuvant, maintenance

\section{Abbreviations:}

TMZ $=$ temozolomide, $\mathrm{WHO}=$ World Health Organization, EORTC $=$ European Organization for Research and Treatment of Cancer, NCIC $=$ National Cancer Institute of Canada, RT $=$ radiotherapy, MRI $=$ magnetic resonance imaging, IMRT $=$ image modulated radiotherapy, $\mathrm{CT}=$ computed tomography, $\mathrm{GCG}=$ giant cell glioblastoma, $\mathrm{GBM}=$ glioblastoma multiforme, $\mathrm{OS}=\mathrm{over}$ all survival TTP $=$ time to tumor progression PFS = progression-free survival

\section{INTRODUCTION}

Glioblastoma (WHO grad IV) is the most frequent and most aggressive primary malignant tumor in adult central nervous system.

Despite multimodal treatment the prognosis remains grim, with median survival of 12-15 month since diagnosis $(1,2)$.

Unfortunately, only $3-5 \%$ of patients are surviving 3 years or more $(3,4)$. In many cases, the reasons of discordant results remain unknown.

Standard care for new glioblastoma diagnosed patient, was established by European Organization for Research and Treatment of Cancer (EORTC)/ National Cancer Institute of Canada (NCIC), published in 2005:
- Radiotherapy (RT): fractionated focal irradiation, 2 Gy per fraction given once daily 5 days per week over 6 weeks (total dose of 60 Gy).

- Concomitant chemotherapy: temozolomide $75 \mathrm{mg} / \mathrm{m}^{2}$ daily, from the first day of radiotherapy until the last day (no longer than 49 days).

- Adjuvant Temozolomide: 6 cycles of 5-day schedule every 28 days; $150 \mathrm{mg} / \mathrm{m}^{2}$ for the first cycle and was increased to $200 \mathrm{mg} / \mathrm{m}^{2}$ beginning with the second cycle (2) .

Maximal surgery of the tumor improves glioblastoma patients prognosis, and this became possible by introducing intra-operator ultrasonography, neuro-navigation and intra-operator coloration. 
Temozolomidum (TMZ) is an alkylating agent which links with DNA molecule and is afecting DNA replication/transcription and is leading to apoptosis (5).

TMZ has the ability to cross blood-brain barrier and to be spontaneously transformed in active metabolite inside of central nervous system (6).

One of the mechanisms involved in TMZ resistance of glioblastoma is increased expression of O6-methyl-guanine DNA methyltransferase, - a DNA reparation protein through alkyl group removal from O6 position of guanine - a target situs for alkylating cytotoxic agents.

We present here 2 new glioblastoma diagnosed cases, which received more than 6 cycles of chemotherapy with TMZ after surgery and standard radiochemistry treatment.

\section{MATERIALS AND METHODS}

\section{Case report 1}

The patient was a 69 -year-old female with medical history of hypertension who was admitted to the hospital with headache, dizziness, and confusion.

Her clinical examination and neurologic exam was almost normal. Blood laboratory data were within normal range.

A brain magnetic resonance imaging (MRI) was performed on February $24^{\text {th }} 2015$ and showed a mass measuring 50,1/47,8/43 $\mathrm{mm}$ within the right frontal lobe, with significant vasogenic edema, seen as a contrast enhancing heterogeneous mass with solid, cystic, and necrosis areas.

On February $26^{\text {th }} 2015$, she underwent a right frontal craniotomy with maximal safe resection of the tumor.

Her postsurgical course unfolded without complications.

Histological examination showed the neoplasia to be a giant cells glioblastoma.

After 4 weeks from surgery, the patient underwent adjuvant radiotherapy using image modulated radiotherapy (IMRT) technique in a conventional fraction schedule, 2 Gy per fraction daily over 6 weeks up to a total dose of $60 \mathrm{~Gy}$ concurrent chemo-
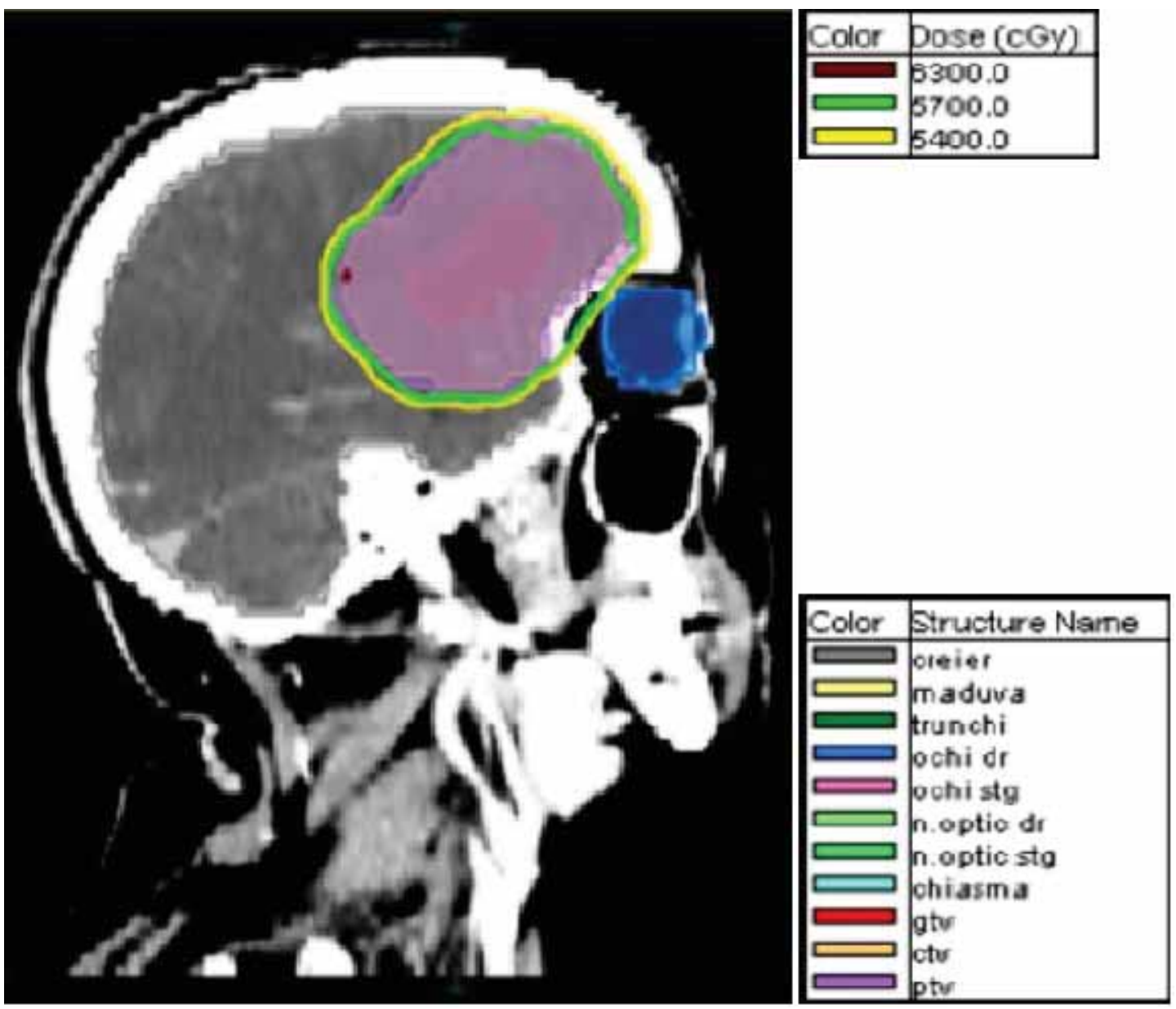

FIGURE 1.

isodose distribution. 

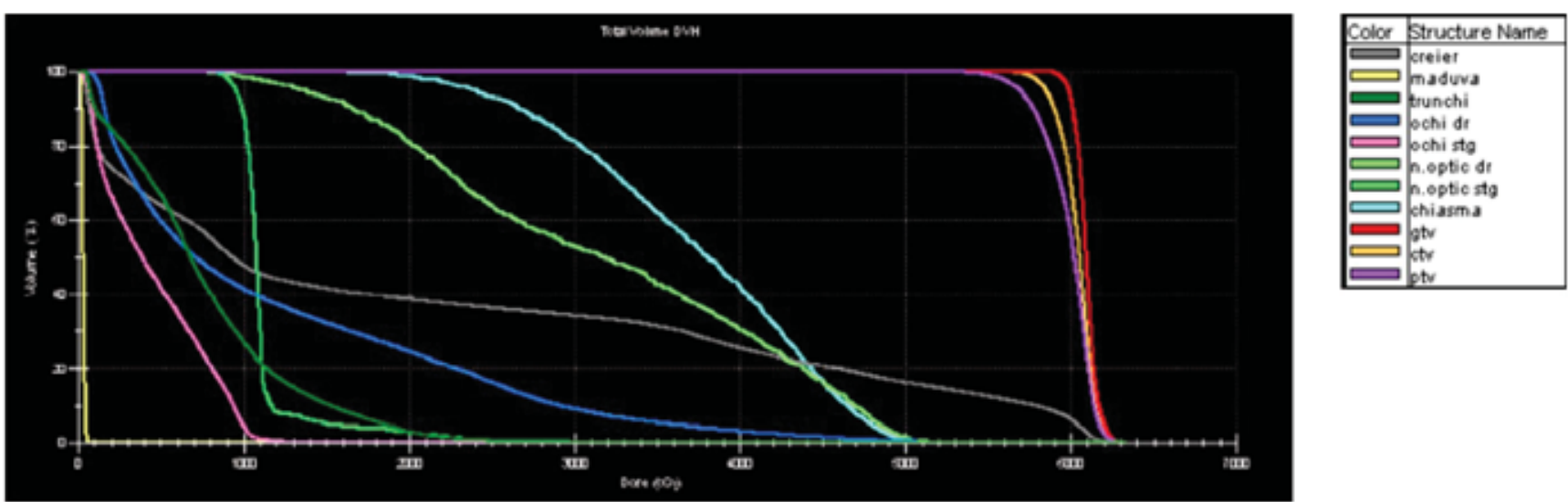

FIGURE 2. Dose-volume histiogram

therapy with TMZ $\left(75 \mathrm{mg} / \mathrm{m}^{2}\right.$ daily. from the first day of radiotherapy until the last day) was administrated Fig. 1,2.

One month following chemotherapy and radiotherapy, the patient was neurologically evaluated, and she performed a cerebral MRI, and there were no signs of disease recurrence. Chemotherapy was continued with Temozolomide $150 \mathrm{mg} / \mathrm{sqm}$ days $1-5$ ( $1^{\text {st }}$ cycle $), q 4 w$, followed by $200 \mathrm{mg} / \mathrm{sqm}$ days 1-5 (for 2 years) without essential toxicity. Last chemotherapy cycle was in 2017, March. She was followed-up for one year (neurological evaluation, MRI, blood tests every 4 months) without any sign of tumor relapse.

In 2018, March, 7th, MRI evaluation showed a relapse, a new tumor in the same area as the previous one and another lesion in left capsulo-lenticular area with a mass effect and its size was 30/25/16 $\mathrm{mm}$.

The multidisciplinary team decided for reirradiation and reintroduction of chemotherapy. Therefore, the patient underwent 2 cycles of chemotherapy with Temozolomide, but due to very unfavorable evolution, the radiotherapy did not start. On 2018, May $5^{\text {th }}$, the patient deceased.

\section{Case report 2}

This case discusses a 38-year-old woman presenting with progressive intracranial hypertension syndrome.

Brain MRI revealed a tumor in the right frontal cerebral lobe, measuring 45/37/41 mm, associated with midline deviation with minimal mass effect and perilesional edema.

A radical surgical removal of the tumour was done in 2016, January $29^{\text {th }}$.

A CT of the head was done in the immediate post-operative period, and it didn't revealed any trace of residual tumor.

After one month after surgery, there was performed a computer tomography (CT) simulation of the head, and it revealed 2 subcortical tumoral nodes in the proximity of resection cavity, measuring 20 and $12 \mathrm{~mm}$.

Our patient also underwent adjuvant chemoradiotherapy with temozolomide in the Stupp regimen (Fig. 3).

The adjuvant chemotherapy was started in 2016, may, $17^{\text {th }}$ with:Temozolomidum $150 \mathrm{mg} / \mathrm{sqm}$ days $1-5$ ( $1^{\text {st }}$ cycle $), q 4 w$, followed by $200 \mathrm{mg} / \mathrm{sqm}$ days
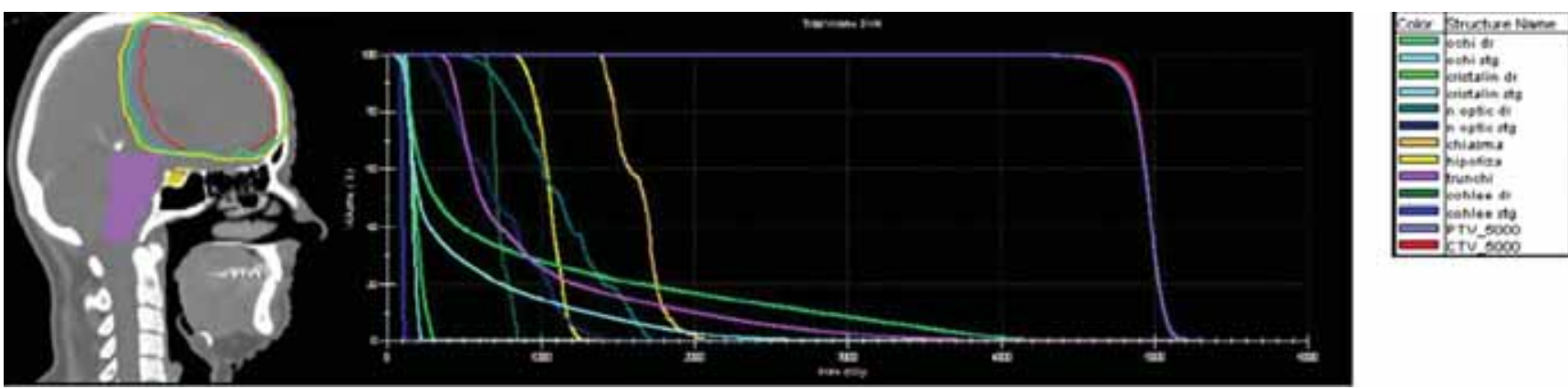

FIGURE 3. Isodose Distribution and Dose volume histiogram 
1-5 until the present (27 months) with good tolerability, with no side effects that could decrease dose or stop the treatment.

The latest MRI review 2018, February $15^{\text {th }}$, describes a single lesion near the resection cavity measuring $3 / 2 \mathrm{~mm}$ axial diameters.

\section{DISCUSSIONS}

In a randomised phase III study comparing concurrent chemoradiotherapy with TMZ and adjuvant six cycles TMZ $150-200 \mathrm{mg} / \mathrm{m}^{2}$ every four weeks, Stupp R et al., showed increased median overall survival (OS) of 14.6 months compared 12.1 months in the RT only arm. The two-year survival rate was $26.5 \%$ with radiotherapy plus Temozolomide and $10.4 \%$ with radiotherapy alone (1).

The 5 years survival rate was $9.8 \%$ in RT with TMZ group, compared to $1.9 \%$ in RT alone group, showing a long-term benefit for the combination treatment (7).

Several changes are being attempted to further improve survival over that conferred standard protocol: extending the adjuvant TMZ doses, use daily metronormic doses or adding new cytotoxic agent.

According to the WHO classification of brain tumors giant cell glioblastoma (GCG) is an uncommon subtype of glioblastoma multiforme accounting for $1-5 \%$ of them (8-10).

GCG tended to occur in younger patients the mean age is 51 years, 11 years less than glioblastoma multiforme (GBM) (8).

Some authors GCG are considering usually have a better prognosis than GBM with longer mean survival rates (11 months compared to 8 months in GBM) and also long-term survival (5 years) is more frequent in GCG $(12,3 \%)$ than GBM $(3,4 \%)(8,11-14)$.

The combination of factors such as younger age at presentation, extension of the surgical resection and the immune histochemical and genetic differences are suggested to be responsible for longer survival.

Studies have shown that extent of resection has prognostic importance, patients without residual tumor survived longer (16.7 versus $11.8 \mathrm{mo}$, $\mathrm{P}<0.0001$ ) (15).

Extensive and complete surgical resection of GBM though representing the most effective way to increase survival of GBM patients but this goal is still difficult to achieve because these tumors are invasive, and are most often located, in neurologically eloquent areas or with extension to these areas (16).

However, neurologic function should not be compromised by surgical efforts at resection, because the Karnofsky performance index remains an important prognostic factor.

The decision to extend the chemotherapy with TMZ for more than 6 cycles, was mainly taken by patiens and their relatives, and also by neurosurgeons and oncologists, as a result of neurological evaluation of toxicity and tumor response to TMZ.

Extended adjuvant therapy was not associated with increased toxicity for the patients we discussed about.

The treatment started with dexamethasone for vasogenic edema and levetiracetam for seizure prophylaxis.

Extended TMZ use more than 6 standard adjuvant cycles is preferred by an increasing number of oncologists.

Several retrospective nonrandomized studies have evaluated the positive impact of long-term use of TMZ on progression free survival(PFS) and over all survival (17-21).

Seiz and colleagues published a study with 114 patients in a single institution who received the standard of treatment (surgery-total resection, subtotal resection or biopsy, radiotherapy and concomitant Temozolomide) followed by adjuvant TMZ for stable and responsive patients.

Number of adjuvant TMZ cycles ranged from 1-57 (received a median of 6 cycles).

OS in all patients was 15 months (95\% CI: 1318 months). Time to tumor progression (TTP) and OS directly correlate with the amount of chemotherapy cycles (each $\mathrm{P}<0.0001$ ) (17).

Barbagallo GMV et al compared data obtained from the group of patients undergoing long-term TMZ treatment (Group A n=19) with data from patients treated with a standard TMZ protocol (Group $\mathrm{B} \mathrm{n}=18$ ).

The patients treated with more than $6 \mathrm{TMZ}$ cycles had OS and PFS that was significantly longer than patients receiving standard treatment (median OS 28 months vs 8 months, respectively; $\mathrm{p}=0.0001$; median PFS 20 months vs 4 months, respectively; $\mathrm{p}=0.0002)$ (18). 
Another study mention it is not known whether the prolongation of the adjuvant phase has a favorable effect on survival (22)

\section{CONCLUSIONS}

Despite advances in the past decade, glioblastoma remains an incurable disease characterized by rapid evolution and resistance to intensive care. Temozolomide is the first and only chemotherapeutic agent that proved its efficacy in the treatment of glioblastoma until now.
Future studies will have to bring further clarifications regarding the OS.

Extended TMZ use more than 6 standard adjuvant cycles remains an open subject.

\section{Source of founding}

This work received financial support through the project entitled "CERO - Career profile: Romanian Researcher", grant number POSDRU/159/ 1.5/S/135760, co-financed by the European Social Fund for Sectoral Operational Programme Human Resources Development 2007-2013.

\section{Conflict of interest: none declared}

\section{REFERENCES}

1. Stupp R., Mason W.P., van den Bent M.J. et al. Radiotherapy plus concomitant and adjuvant temozolomide for glioblastoma. N Engl J Med 2005; 352: 987-996. doi: 10.1056/NEJMoa043330.

2. Wen P.Y., Kesari S. Malignant gliomas in adults. N Engl J Med 2008; 359: 492-507. doi: 10.1056/NEJMra0708126.

3. Ohgaki H. Epidemiology of brain tumors. Methods Mol Biol 2009; 472: 323-342. doi: 10.1007/978-1-60327-492-0_14.

4. Martinez R., Schackert G., Yaya-Tur R. et al. Frequent hypermethylation of the DNA repair gene MGMT in long-term survivors of glioblastoma multiforme. J Neurooncol 2007; 83: 91-93. doi: 10.1007/s11060-006-9292-0.

5. Fu D., Calvo J.A., Samson L.D. SERIES: Genomic instability in cancer Balancing repair and tolerance of DNA damage caused by alkylating agents. Nat Rev Cancer. 2012; 12(2):104-120. doi:10.1038/ nrc3185.

6. Agarwala S.S., Kirkwood J.M. Temozolomide, a novel alkylating agent with activity in the central nervous system, may improve the treatment of advanced metastatic melanoma. Oncologist. 2000; 5(2):144-151. doi: 10.1634/theoncologist.5-2-144.

7. Stupp R., Hegi M.E., Mason W.P. et al. Effects of radiotherapy with concomitant and adjuvant temozolomide versus radiotherapy alone on survival in glioblastoma in a randomised phase III study: 5-year analysis of the EORTC-NCIC trial. Lancet Oncol. 2009; 10(5):45966. doi: 10.1016/S1470-2045(09)70025-7

8. Kozak K.R., Moody J.S. Giant cell glioblastoma: a glioblastoma subtype with distinct epidemiology and superior prognosis. Neuro Oncol. 2009; 11(6):833-41. doi: 10.1215/15228517-2008-123

9. Palma L., Celli P., Maleci A. et al. Malignant monstrocellular brain tumours. A study of 42 surgically treated cases. Acta Neurochir (Wien). 1989; 97(1-2):17-25

10. Deb P., Sharma M.C., Chander B. et al. Giant cell glioblastoma multiforme: report of a case with prolonged survival and transformation to gliosarcoma. Childs Nerv Syst. 2006; 22(3):314-9. doi: 10.1007/ s00381-005-1239-9

11. Prayson R.A. Histologic classification of high-grade gliomas. In: Barnett $\mathrm{GH}$, editor. High-grade gliomas diagnosis and treatment. Totowa, NJ: Humana Press; 2007. p. 3-36. doi: 10.1007/978-159745-185-7_1

12. Aldape K.D., James C.D. Pathology and molecular classification. In: Bernstein M, Berger MS, editors. Neurooncology: the essentials. $2^{\text {nd }}$ ed. New York: Thiueme; 2008.p. 18-31.
13. Klein R., Mölenkamp G., Sörensen N. et al. Favorable outcome of giant cell glioblastoma in a child. Report of an 11-year survival period. Childs Nerv Syst.1998;14(6):288-91. doi: 10.1007/s003810050228

14. Folgueral J.M.V., Mascarenhas L., Costa J.A. et al. Giant cell glioblastoma: review of the literature and illustrated case. Neurocirugía (Astur). 2008;19:343-349

15. Stummer W., Reulen H.J., Meinel T. et al. Extent of resection and survival in glioblastoma multiforme: identification of and adjustment for bias. Neurosurgery. 2008 ;62(3):564-76. doi: 10.1227/01. NEU.0000346231.57554.44

16. Wilson T.A., Karajannis M.A., Harter D.H. Glioblastoma multiforme: State of the art and future therapeutics. Surg Neurol Int. 2014; 5:64. doi: 10.1001/archneur.1964.00460230112012

17. Seiz M., Krafft U., Freyschlag C.F. et al. Long-term adjuvant administration of temozolomide in patients with glioblastoma multiforme: experience of a single institution. J Cancer Res Clin Oncol 2010; 136:1691-5. doi: 10.1007/s00432-010-0827-6

18. Barbagallo G.M., Paratore S., Caltabiano R. et al. Long-term therapy with temozolomide is a feasible option for newly diagnosed glioblastoma: a single-institution experience with as many as 101 temozolomide cycles. Neurosurg Focus 2014; 37:E4. doi: 10.3171/2014.9.FOCUS14502

19. Rivoirard R., Falk A.T., Chargari C. et al. Long-term results of a survey of prolonged adjuvant treatment with temozolomide in patients with glioblastoma (SV3 Study). Clin Oncol (R Coll Radiol) 2015; 27:486-7. doi: 10.1016/j.clon.2015.04.003

20. Darlix A., Baumann C., Lorgis V. et al. Prolonged administration of adjuvant temozolomide improves survival in adult patients with glioblastoma. Anticancer Res 2013; 33:3467-3474.

21. Rold an Urgoiti G.B., Singh A.D., Easaw J.C. Extended adjuvant temozolomide for treatment of newly diagnosed glioblastoma multiforme. J Neurooncol 2012; 108:173-177. doi: 10.1007/ s11060-012-0826-3

22. Blumenthal D.T., Stupp R., Zhang P. et al. The impact of extended adjuvant temozolomide in newly diagnosed glioblastoma: $A$ secondary analysis of EORTC and NRG Oncology/RTOG. Neuro Oncol 2015; 17(Suppl 5):v2. doi: 10.1093/neuonc/nov206.08 